\title{
Density Estimation is Influenced More by Mass When Objects are Denser
}

\author{
Lara Merken $^{1}$ and Vonne van Polanen ${ }^{2(\varpi)}$ \\ 1 Department of Neurosciences, KU Leuven, Herestraat 49, 3000 Leuven, Belgium \\ lara.merken@kuleuven. be \\ 2 Department of Movement Sciences and Leuven Brain Institute, \\ KU Leuven, Tervuursevest 101, 3001 Leuven, Belgium \\ vonne.vanpolanen@kuleuven. be
}

\begin{abstract}
When judging the heaviness of objects, the perceptual estimate can be influenced by the object's density next to its mass. In the present study, we investigated whether density estimates might be similarly affected by object mass. Participants lifted objects of different sizes and masses in a virtual reality environment and estimated the density. We found that density perception was influenced both by density and mass, but not for the lowest density value, which could be perceived correctly. A modelling procedure on fitted slopes through the different objects revealed that density contributed $56 \%$ to the density estimate. However, if low- and high-density values were modelled separately, contributions of $100 \%$ and $41 \%$ were found for the low and high densities, respectively. These results indicate that perception of heaviness and density are closely related but can be better distinguished with objects of lower density and mass.
\end{abstract}

Keywords: Perception $\cdot$ Density $\cdot$ Mass $\cdot$ Heaviness

\section{Introduction}

When we manipulate objects, we can perceive object properties such as size, weight and material. During object lifting, we receive haptic feedback that allows us to make a heaviness estimation of the object. The brain defines the most optimal estimate (i.e. with minimal variance) based on different sensory sources, such as visual and haptic information [5]. In other words, a combination of cues can be used to make a perceptual estimate. Regarding heaviness perception, one of these cues might be object density, which is the relation between the size and weight of the object and depends on the objects' material.

Previous research has shown that density might contribute to heaviness perception. Five decades ago, Ross and Di Lollo [12] suggested that the impact

Research supported by a Fonds Wetenschappelijk Onderzoek grant to VVP (FWO post-doctoral fellowship, Belgium, 12X7118N). The authors would like to thank Ellen Vervoort for her help in data collection.

(C) The Author(s) 2020

I. Nisky et al. (Eds.): EuroHaptics 2020, LNCS 12272, pp. 87-95, 2020.

https://doi.org/10.1007/978-3-030-58147-3_10 
of mass and density in heaviness estimation shifts with the objects' density. The authors claimed that heaviness estimations of low-density objects were predominantly based on mass, while for high-density objects the density had more impact. In another study was suggested that the combination of density and mass controls heaviness discrimination. When both density and mass simultaneously increase/decrease, heaviness perception is more accurate. In contrast, accuracy declines when the factors change in opposite directions [9]. More recently, it was proposed that heaviness estimation is formed from a weighted combination of mass and density information, depending on the properties' reliability $[3,13]$.

The inclusion of other object properties, such as density, into heaviness estimation might provide an explanation for some weight-related illusions. The sizeweight illusion [1] shows that a smaller object is perceived as being heavier than a larger object with an identical mass, suggesting that the unexpected denser object feels heavier. Similarly, the material-weight illusion shows that visual features of the material bias the perception of heaviness. When two objects of seemingly different materials but equal size and mass are presented, the denserlooking material (i.e. metal vs wood) is perceived to be lighter [4].

These studies show that density influences heaviness perception. It has also been observed that density estimations can be influenced by object mass [7], suggesting that neural processing of these concepts is related. However, in that study, mass was not varied. Therefore, it has not been systematically examined how mass could affect density perception. Object density cannot be directly perceived but has to be inferred from both the size and mass of an object, using visual and/or haptic information. The perception of density is important to distinguish between different object materials, such as wood or metal, or, e.g. determine the content of a closed box. For this purpose, further research on density perception might give new insights into object perception. In the present study, we investigated how participants perceived the density of objects of different sizes and weights. Participants lifted objects in a virtual reality set-up and judged the density. We found that the mass of objects affected the density estimate but more so when objects were denser and heavier.

\section{Methods}

\subsection{Set-Up and Procedure}

12 participants took part in the study (age range 18-26 years, 6 females, 11 selfreported right-handed). They had normal or corrected-to-normal vision and had no known neurological impairments. Before the experiment they all signed an informed consent form. The study was approved by the local ethical committee of KU Leuven.

The experiment was performed in a virtual reality (VR) setup that simulated virtual cuboids on a checkerboard background. The visual virtual environment was projected with a 3D screen (Zalman) on a mirror, under which participants moved their hands so they were unable to see their hands. The tips of the thumb and index finger were inserted into two haptic force-feedback devices (Phantom 
Premium 1.5, Sensable) to be able to haptically simulate the objects. The fingertips were visually indicated by two red spheres on the screen. More details of the VR setup can be found in [11]. Forces were measured in 3 directions and sampled at a $500 \mathrm{~Hz}$ frequency. 12 cuboids were used in the experiment with different size-mass combinations (Table 1 ). The size was varied by changing object height, while keeping the width and depth at $5 \mathrm{~cm}$. Each density value $(0.75$, $1.25,1.75,2.25 \mathrm{~g} / \mathrm{cm}^{3}$ ) was presented in 3 different size-mass combinations.

Participants were seated in front of the VR setup and familiarised with the environment. In each trial, a virtual object appeared and participants had to grasp it after hearing a beep. They lifted the object with thumb and index finger up to a target height, indicated with a yellow sphere. After they had replaced the object, they were asked to estimate the density of the object on a self-chosen scale. Before the experiment, they received an explanation of the concept of density and were instructed not to report the heaviness, but only the density of the object. Participants were unaware of the number of objects or densities used in the experiment. Each object was presented 10 times, giving a total of 120 trials, which were presented in a randomised order. Two objects of the same weight or size were never presented in a row, so participants could not compare the density of two sequential objects by only varying one parameter. The experiment was divided into two sessions with a short break in between.

Table 1. Mass $(\mathrm{g})$, size $(\mathrm{cm})$ and density $\left(\mathrm{g} / \mathrm{cm}^{3}\right)$ of the objects used in the experiment.

\begin{tabular}{l|l|l|l|l|l|l}
\hline Size/Mass & 112.5 & 157.5 & 202.5 & 262.5 & 337.5 & 472.5 \\
\hline 3.6 & 1.25 & 1.75 & 2.25 & & & \\
\hline 6 & 0.75 & & & 1.75 & 2.25 & \\
\hline 8.4 & & 0.75 & & 1.25 & & 2.25 \\
\hline 10.8 & & & 0.75 & & 1.25 & 1.75 \\
\hline
\end{tabular}

\section{$2.2 \quad$ Analysis}

The individual density estimates of participants were normalised to z-scores. The normalised estimates were averaged over the trials for each object. Missing samples $(0.03 \%)$ of the force data were interpolated. Next, force data was filtered with a $2^{\text {nd }}$ order low-pass Butterworth filter with a cut-off frequency of $15 \mathrm{~Hz}$. The load forces (LF) were the sum of the vertical forces and were used to calculate the loading phase duration (LPD). We used the LPD as a measure of lifting performance, since this is indicative of force planning and different for different object masses [8]. The LPD was calculated as the time between LF onset $(\mathrm{LF}>0.1 \mathrm{~N})$ and lift-off ( $\mathrm{LF}>$ object weight). 31 (2\%) trials were removed from the force analysis due to incorrect lifts.

A linear mixed model was used to statistically determine whether density and mass affected the perceived density and LPD. The z-scored estimates or the 
LPD was the dependent variable, density and mass were included as fixed factors and participant number as random factor. The density $\times$ mass interaction and the intercepts were also included in the model. We used maximum likelihood for the estimation and a $1^{\text {st }}$ order autoregressive model. Post-hoc comparisons were performed with a Bonferroni correction. The alpha was set to 0.05.

The linear mixed model provides estimates for each density and mass, because they were included as fixed factors, but no relative contribution of those factors could be computed. Therefore, we performed a different modelling procedure where we assumed that density estimates only relied on mass and density. The weights given to density and mass were $w_{d}$ and $w_{m}$, respectively, and $w_{d}+w_{m}=$ 1. In general, a relation such as $D=w_{d} a d+w_{m} b m+c$ can be expected, where $D$ is the estimated density, $d$ and $m$ the density and mass values and $a, b$ and $c$ are constants. Since we only had one data set, we could not fit all the unknown constants and the weights. Therefore, we chose to fit the relation between $\mathrm{D}$ and $d$ or $\mathrm{m}$ by calculating the slopes: $s_{d}$ and $s_{m}$, respectively. We assumed that the slopes could be seen as a relative value of a constant value, which we called the maximum slope:

$$
\begin{aligned}
& s_{m}=w_{d} s_{\max , m} \\
& s_{d}=\left(1-w_{d}\right) s_{\max , d}
\end{aligned}
$$

More specifically, to assess the contribution of density to the density estimates, we plotted the density estimates against the mass of the objects (Fig. 1). Through similar densities, we fitted linear regressions to obtain 4 slopes ('density slopes'). When solely relying on density, slopes will be equal to 0 (Fig. 1A). However, if density would be ignored and participants rely on mass, the slopes would be on the line between the lowest and highest mass (Fig. 1B), i.e. the maximum slope $\left(s_{\max , d}\right)$. The slopes will have values in between these cases when participants have a balanced weighting of mass and density. Similarly, to evaluate the contribution of mass, 6 slopes could be fitted to objects with equal mass ('mass slopes') when density estimates are plotted against object density. If participants rely solely on density, mass slopes will be maximal (Fig. 1A). However, if they ignore density and only rely on mass, mass slopes are 0 (Fig. 1B).

We set the maximum slopes as the differences between the estimate for the object with the lowest mass-density combination and the highest mass-density combination: $s_{\max , d}=6.13$ and $s_{\max , m}=1.47$. Next, the only free parameter, $w_{d}$, was obtained by minimizing the sum of the squared errors between the modelled slopes from Eq. 1 and the measured slopes from the data. Note that only slopes were fitted to estimate $w_{d}$ and not density estimates. Therefore, the modelled slopes and weights cannot be used to calculate expected density estimates from the mass and density of objects. The plotted modelled slopes in the Fig. 1 and 2 only serve to compare the slope value, as the intercepts were not fitted, but estimated by dividing the maximum slope into equal steps for the number of slopes and depended on $w_{d}$. 

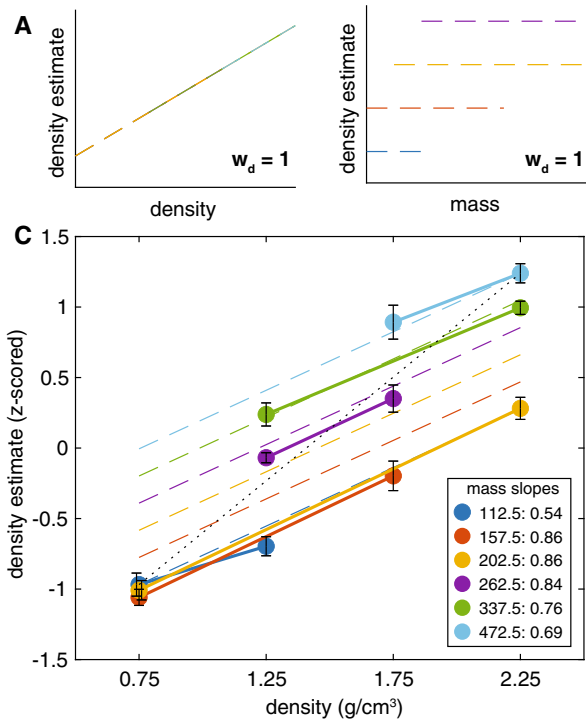
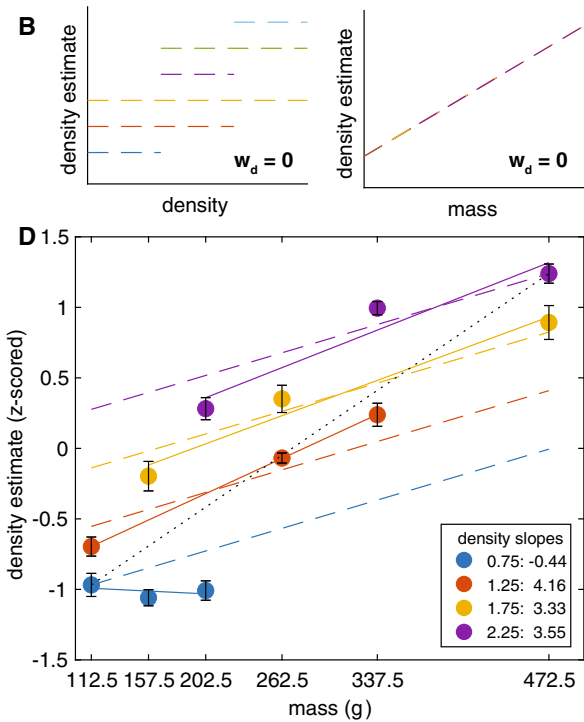

Fig. 1. Mass and density slopes. A-B: predicted slopes if the weight given to density $\left(w_{d}\right)$ is equal to 1 or 0 . C-D: mean density estimates (solid circles) and standard errors (error bars). Solid lines represent measured slopes (C: mass, D: density) fitted to the data, which values are indicated in the legend. Dashed and dotted lines indicate modelled slopes and maximum slopes from the model.

\section{Results}

Figure 1 shows the average density estimates for each object. It can be seen that the estimates increase both with density and mass. The linear mixed model on the density estimates revealed significant effects of mass $(F(3,134)=45, p<$ $0.001)$ and density $(F(5,107)=97, p<0.001)$, but not their interaction $(p=$ $0.533)$. Post-hoc effects showed that all density values differed significantly from each other (all $p<0.001$ ). The masses all differed significantly from each other (all $p<0.027)$, except the two lowest masses $(p=0.208)$. Thus, for most objects, both mass and density affected the perceptual estimate for density.

For LPD, there was only a significant effect of mass $(F(5,114)=55, p<$ $0.001)$. Except for the $157.5 \mathrm{~g}$ and $202.5 \mathrm{~g}$ objects $(p=0.72)$, and the $202.5 \mathrm{~g}$ and $262.5 \mathrm{~g}$ objects $(p=0.75)$, all other masses differed from each other (all $p<0.003)$. The effect of density or the interaction of density $\times$ mass were not significant, indicating that lifting performance was only affected by object mass, not density. Therefore, the density estimates for different densities could not be explained by alterations in lifting performance.

The measured slopes through objects with similar mass or density are shown in Fig. 1 and values are displayed in the legend. One sample t-tests of participants' slopes indicated that all mass slopes were significantly different from zero (all $p<0.004$ ). The density slopes were also significantly different from zero 
$(p<0.001)$, except for the lowest density $(p=0.75)$. The density and mass slopes were used to determine the contribution of density and mass to the density estimates by modelling one slope value for the density slopes and one for the mass slopes. The modelled slopes were 2.67 and 0.83 for density and mass slopes, respectively (Fig. 1). We found a $w_{d}$ of 0.56 , which explained $39 \%$ of the data ( $R^{2}$ value).

Although most modelled slopes seem similar to measured slopes, the fits do not seem optimal for the lowest masses and the lowest density, which could clarify the low explained variance. Therefore, the modelling procedure was repeated, but now not all slopes were assumed to be the same: $w_{d}$ was split between the lowest and the other three density slopes, and between the three lower and three higher mass slopes. For the mass slopes, new separate maximum slopes were calculated, since now only half of the range was covered for low and high values: $s_{\max , m, l}=0.83, s_{\max , m, h}=1.31$. The original $s_{\max , d}$ was used for the density slopes. For this model, we found $w_{d, l}=1$ and $w_{d, h}=0.41$. The explained variance was $96 \%$ and the results are shown in Fig. 2. Here it is seen that all modelled slopes are similar to the measured density slopes.

The procedure was also performed on the individual data. A range of weights was found with $w_{d}$ ranging between $0.12-1.0($ mean $=0.55)$, with the explained variance ranging from $0-97 \%$. However, if we also split the fits for the lower and higher densities, we obtained weights of $w_{d, l}$ between $0.06-1.0$ and $w_{d, h}$ between $0.04-1.0$, with means of 0.78 and 0.43 , respectively. For the low density, $8 / 12$ participants had a $w_{d, l}>0.98$, and for the high densities, $11 / 12$ participants had a $w_{d, h}<0.54$. Here, the explained variance was between $11-97 \%$, with $>50 \%$ and $>90 \%$ in 10/12 and 6/12 participants, respectively. All in all, it seems that the weight that was assigned to density for density judgements varied among participants but that most participants gave higher weights to density for low densities, whereas for higher densities, the weight assigned to mass was higher.

\section{Discussion}

In this study, we investigated how humans perceive density when presented with objects of different size and mass. We found that density estimates depended both on density and mass. This is similar to previous studies that found that heaviness estimates depended on these two object properties as well $[3,13]$ and the finding that participants had more difficulties reporting a difference in heaviness with objects of different compared to equal densities [9]. Hence, density and heaviness perception might be closely related.

We found a contribution of $56 \%$ of density relative to mass for density estimates. This seems slightly different from the density weight of $29 \%$ for heaviness estimation found in [13], suggesting that the weighing of density and mass can be adapted to the required percept. However, it must be noted that the contribution varied for different densities and individuals.

Interestingly, we found that the weight of mass on density perception increased with density, indicating that cue weighing can also depend on intensity. Whereas density estimates were not affected by mass for the lowest density, 

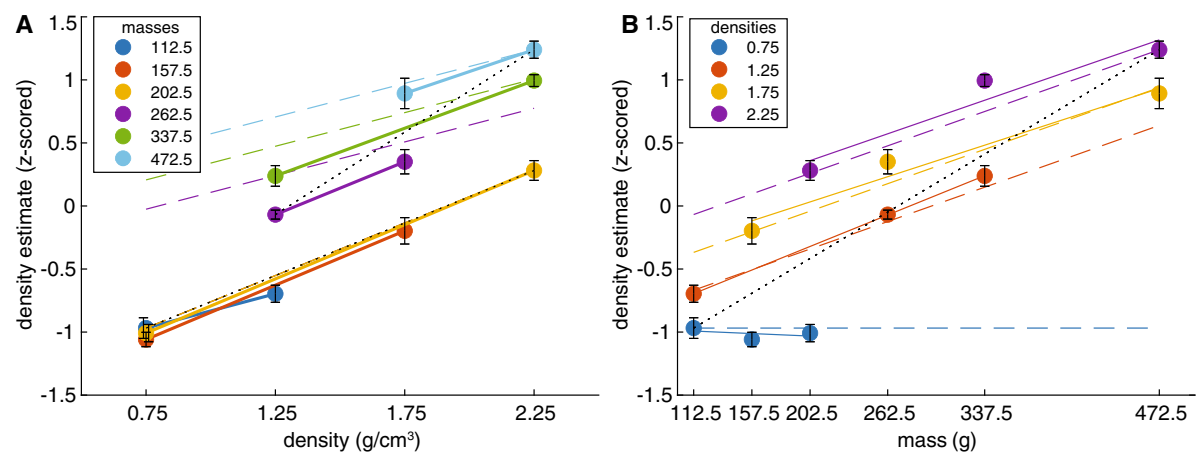

Fig. 2. Results for the mass (A) and density slopes (B) for low and high values separately. Solid circles and error bars represent density estimates mean and standard errors, respectively. Solid and dashed lines represent fitted and modelled slopes, respectively. The black dashed lines are the maximum slopes used in the model.

the influence of mass became more pronounced for objects with higher densities. In our experiment, the low-density objects all had the lowest masses as well. Therefore, it is also possible that mass influences density estimates more when objects are heavier. At first hand, a bias of mass seems logical, since in daily life we might be more familiar with judging object mass than density. However, this does not explain why low-density objects can be perceived accurately. Possibly, we are more familiar with handling objects of low densities, such as wood or plastic, and have more experience in distinguishing such objects. Furthermore, in daily life, we experience a non-linear distribution of size and weight. These learned statistics of everyday objects can be used to estimate those of novel objects. In general, small objects are seen as denser in comparison with objects larger in size, since in real life small objects tend to be denser [10]. Similarly, daily life experience might also suggest a relation between heavy and dense objects. Finally, for heaviness estimations, lower contributions of density were found for objects with lower densities and mass [12], further suggesting that for low densities and light objects, the object properties density and mass can be better disentangled.

The mutual influence of density and mass in object perception suggest that brain processes for these properties are related. A functional magnetic resonance imaging study revealed that object density is processed in a higher-order area of the brain (left ventral premotor area) and the primary motor cortex encodes object weight [2]. Density and mass perception might mutually influence each other since these areas are closely connected [6]. Therefore, it could be difficult for humans to estimate these properties independently.

The present study also has some limitations. First, we used a limited set of object sizes and weights for each density. Therefore, it is difficult to disentangle whether low densities can be accurately perceived due to the low density or due to low masses. A larger object set would include very small or large objects 
which would have been difficult to grasp in our VR setup. In addition, since we estimated maximum slopes from our data set, the weightings are specific to our data set and the actual values might not be generalised to other data sets, but only the relative weighting between density and mass can be interpreted. Furthermore, it is possible that participants did not fully understand the concept of density and reported mass instead, despite the careful explanation of the density concept to each subject. However, results clearly showed a contribution of density, especially for low-density objects, ruling out a judgement of object mass only. Finally, it must be noted that we used mass and density as the only contributors to the density estimates, although other factors might contribute as well. Particularly object volume cannot be fully distinguished from density, and when volume and mass were used in the model fit, similar results were seen.

In conclusion, both mass and density influence density perception, but mass affects density perception more when objects are denser and heavier. Our study supports the idea that density perception is closely related to heaviness perception, but also indicates that the weighing of these properties can vary based on their intensity. The perception of density is important for distinguishing objects of different materials or objects with different contents. This could be relevant when designing virtual environments or controlling robotic arms to interact with different objects of different materials. The interaction of density and mass in object perception should be taken into account.

\section{References}

1. Charpentier, A.: Analyse experimentale de quelques elements de la sensation de poids. Archive de Physiologie normale et pathologiques 3, 122-135 (1891)

2. Chouinard, P.A., Large, M.E., Chang, E.C., Goodale, M.A.: Dissociable neural mechanisms for determining the perceived heaviness of objects and the predicted weight of objects during lifting: an fMRI investigation of the size-weight illusion. NeuroImage 44(1), 200-12 (2009)

3. Drewing, K., Bergmann Tiest, W.M.: Mass and density estimates contribute to perceived heaviness with weights that depend on the densities' reliability. In: 2013 World Haptics Conference, WHC 2013, vol. 2, pp. 593-598. IEEE (2013)

4. Ellis, R.R., Lederman, S.J.: The material-weight illusion revisited. Percept. Psychophys. 61(8), 1564-1576 (1999)

5. Ernst, M.O., Banks, M.S.: Humans integrate visual and haptic information in a statistically optimal fashion. Nature 415(6870), 429-433 (2002)

6. Grafton, S.T.: The cognitive neuroscience of prehension: recent developments. Exp. Brain Res. 204(4), 475-491 (2010)

7. Huang, I.: The size-weight illusion and the weight-density illusion. J. General Psychol. 33, 65-84 (1945)

8. Johansson, R.S., Westling, G.: Coordinated isometric muscle commands adequately and erroneously programmed for the weight during lifting task with precision grip. Exp. Brain Res. 71, 59-71 (1988)

9. Kawai, S.: Heaviness perception. II. Contributions of object weight, haptic size, and density to the accurate perception of heaviness or lightness. Exp. Brain Res. 147(1), 23-8 (2002) 
10. Peters, M.A.K., Balzer, J., Shams, L.: Smaller = denser, and the brain knows it: natural statistics of object density shape weight expectations. PloS One 10(3), e0119794 (2015)

11. van Polanen, V., Tibold, R., Nuruki, A., Davare, M.: Visual delay affects force scaling and weight perception when lifting objects in virtual reality. J. Neurophysiol. 121, 1398-1409 (2019)

12. Ross, J., Di Lollo, V.: Differences in heaviness in relation to density and weight. Percept. Psychophys. 7(3), 161-162 (1970)

13. Wolf, C., Bergmann Tiest, W.M., Drewing, K.: A mass-density model can account for the size-weight illusion. PloS One 13(2), e0190624 (2018)

Open Access This chapter is licensed under the terms of the Creative Commons Attribution 4.0 International License (http://creativecommons.org/licenses/by/4.0/), which permits use, sharing, adaptation, distribution and reproduction in any medium or format, as long as you give appropriate credit to the original author(s) and the source, provide a link to the Creative Commons license and indicate if changes were made.

The images or other third party material in this chapter are included in the chapter's Creative Commons license, unless indicated otherwise in a credit line to the material. If material is not included in the chapter's Creative Commons license and your intended use is not permitted by statutory regulation or exceeds the permitted use, you will need to obtain permission directly from the copyright holder.

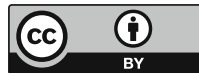

